\title{
Pulsars with the Australian Square Kilometre Array Pathfinder
}

I. H. Stairs ${ }^{\mathrm{a}}$, M. J. Keith ${ }^{\mathrm{b}}$, Z. Arzoumanian ${ }^{\mathrm{c}}$, W. Becker ${ }^{\mathrm{d}}$, A. Berndsen ${ }^{\mathrm{a}}$, A. Bouchard ${ }^{\mathrm{e}}$, N. D. R. Bhat ${ }^{\mathrm{f}}$, M. Burgay ${ }^{\mathrm{g}}$, D. J. Champion ${ }^{\mathrm{h}}$, S. Chatterjee ${ }^{\mathrm{i}}$, T. Colegate ${ }^{\mathrm{j}}$, J. M. Cordes ${ }^{\mathrm{i}}$, F. M. Crawford ${ }^{\mathrm{k}}$, R. Dodson' ${ }^{\text {, P. C. C. Freire }}{ }^{\mathrm{h}}$, G. B. Hobbs ${ }^{\mathrm{b}}$, A. W. Hotan ${ }^{\mathrm{j}}$, S. Johnston ${ }^{\mathrm{b}}$, V. M. Kaspi ${ }^{\mathrm{e}}$, V. Kondratiev ${ }^{\mathrm{m}}$, M. Kramer ${ }^{\mathrm{h}, \mathrm{n}}$, T. J. W. Lazio ${ }^{\circ}$, W. Majid ${ }^{\mathrm{o}}$, R. N. Manchester ${ }^{\mathrm{b}}$, D. J. Nice ${ }^{\mathrm{p}}$, A. Pellizoni ${ }^{\mathrm{g}}$, A. Possenti ${ }^{\mathrm{g}}$, S. M. Ransom ${ }^{\mathrm{q}}$, N. Rea ${ }^{\mathrm{r}}$, R. Shannon ${ }^{\mathrm{b}}$, R. Smits ${ }^{\mathrm{m}, \mathrm{n}}$, B. W. Stappers ${ }^{\mathrm{n}}$, D. F. Torres ${ }^{\mathrm{s}}$, A. G. J. van Leeuwen ${ }^{\mathrm{m}, \mathrm{t}}$, W. van Straten ${ }^{\mathrm{f}}$ and P. Weltevrede ${ }^{\mathrm{n}}$

${ }^{a}$ Dept. of Physics and Astronomy, UBC, 6224 Agricultural Road, Vancouver, BC V6T 1Z1 Canada

${ }^{b}$ CSIRO Astronomy and Space Science, Australia Telescope National Facility, CSIRO, PO Box 76, Epping, NSW 1710, Australia

${ }^{c}$ CRESST/USRA, NASA Goddard Space Flight Center, Code 662, Greenbelt, MD 20771, USA

${ }^{d}$ Max-Planck-Institut fÃijr extraterrestrische Physik, Giessenbachstrasse 1, 85748 Garching, Germany

${ }^{e}$ Department of Physics, McGill University, 3600 University Street, Montreal, Quebec, H3A 2T8, Canada

${ }^{f}$ Swinburne University of Technology, Centre for Astrophysics and Supercomputing, Mail H39, PO Box 218, VIC 3122, Australia

${ }^{g}$ INAF - Osservatorio Astronomico di Cagliari, località Poggio dei Pini, strada 54, I-09012 Capoterra, Italy

${ }^{h}$ Max Planck Institut für Radioastronomie, Auf dem Hügel 69, 53121 Bonn, Germany ${ }^{i}$ Astronomy Department, Cornell University, Ithaca, NY 14853, USA

${ }^{j}$ ICRAR/Curtin Institute of Radio Astronomy, GPO Box U1987, Perth, WA 6845 Australia

${ }^{k}$ Department of Physics and Astronomy, Franklin and Marshall College, P.O. Box 3003, Lancaster, PA 17604, USA

${ }^{I}$ ICRAR/University of Western Australia, Fairway 7, Crawley, Perth, WA 6009 Australia

${ }^{m}$ Netherlands Institute for Radio Astronomy (ASTRON), Postbus 2, 7990 AA Dwingeloo, The Netherlands

${ }^{n}$ University of Manchester, Jodrell Bank Centre for Astrophysics, Alan Turing Building, Manchester M13 9PL, U.K.

${ }^{\circ}$ Jet Propulsion Laboratory, M/S 138-308, 4800 Oak Grove Dr., Pasadena, CA 91109, USA

${ }^{p}$ Department of Physics, Lafayette College, Easton, PA 18042, USA

${ }^{q}$ National Radio Astronomy Observatory, Charlottesville, VA 22903, USA

${ }^{r}$ Institut de Ciències de l'Espai (IEEC-CSIC), Campus UAB, Facultat de Ciències, Torre C5-parell, 2 a planta, 08193 Barcelona, Spain

${ }^{5}$ ICREA \& Institut de Ciències de l'Espai (IEEC-CSIC), Campus UAB, Facultat de Ciències, Torre C5-parell, 2 a planta, 08193 Barcelona, Spain

${ }^{t}$ Astronomical Institute "Anton Pannekoek," University of Amsterdam, 1098 SJ Amsterdam, Netherlands 


\begin{abstract}
The Australian Square Kilometre Array Pathfinder (ASKAP) is a 36-element array with a 30-square-degree field of view being built at the proposed SKA site in Western Australia. We are conducting a Design Study for pulsar observations with ASKAP, planning both timing and search observations. We provide an overview of the ASKAP telescope and an update on pulsar-related progress.
\end{abstract}

Keywords: pulsars, ASKAP

PACS: $95.55 . J z, 95.75 . \mathrm{Wx}, 97.60 . \mathrm{Gb}$

\title{
INTRODUCTION
}

The Australian Square Kilometre Array Pathfinder (ASKAP) is currently being built at the proposed site for the Square Kilometer Array (SKA) in Western Australia. ASKAP will be a 36-element array of 12-m antennas with baselines of up to several $\mathrm{km}$; it is well-described in Deboer et al. [1]. It will use checkerboard-style phased-array feeds [2] with dithering to achieve roughly uniform sensitivity over a field of view (FOV) of 30 square degrees. Several primary beams will be used to cover the full FOV, with the number of beams increasing with frequency so as to maintain the same size FOV at all frequencies between $700 \mathrm{MHz}$ and $1800 \mathrm{MHz}$. Roughly $300 \mathrm{MHz}$ of bandwidth will be available at any time. ASKAP will also offer multiple tied-array beams, steerable within the FOV, for projects interested in point-source work. The projected system temperature is roughly $50 \mathrm{~K}$.

The goals of the ASKAP project are to demonstrate technologies (specifically inexpensive parabolic reflectors and phased-array feeds) relevant to the SKA, to carry out science related to the SKA Key Science Projects [3], to establish a radio astronomy site in Western Australia and to build a user base for the SKA [4].

The choice has been made to optimize the combination of antenna layout, FOV, observing frequencies and system temperature to allow rapid surveys of neutral Hydrogen in our Galaxy and nearby galaxies, along with continuum surveys of galaxies to high redshift. Polarization surveys and exploration of transient phenomena are also priorities. The full science case for ASKAP can be found in Johnston et al. [5, 6].

ASKAP will reduce all imaging data taken with one or more real-time pipelines, archiving images and spectral lines as required. Observers will retrieve data from the archive rather than operating the telescope themselves. All archived data will be publically available [1]. To ensure that good analysis pipelines are developed, observers have been encouraged to organize into "Science Survey" teams, 10 of which have been chosen to carry out "Design Studies" in the period 2009-2011. Advantages of joining a Science Survey Project (SSP) include ongoing communication with and support from CASS during ASKAP development, ensuring that SSP scientists will be well-placed to understand and extract science from the data the moment they appear in the archive.

With a total collecting area similar to that of the Parkes telescope, but significantly higher system temperature, ASKAP is perhaps not the most obvious choice for pulsar observations. Nevertheless, pulsars are at the core of one of the 5 SKA Key Science Projects ("Strong-Field Tests of Gravity Using Pulsars and Black Holes") and form one of the two driving projects for the Phase I SKA. Since pulsar observations have 
historically been carried out primarily using large single dishes, we have formed the COAST ("Compact Objects with ASKAP: Surveys and Timing") SSP collaboration to work on the technical aspects of migrating pulsar observations to interferometers while carrying out niche pulsar science in the areas where ASKAP can excel.

\section{THE PULSAR SCIENCE CASE}

The COAST SSP proposal comprises both search and timing observations, aimed at a broad range of pulsar-related science.

\section{Timing}

Despite somewhat lower point-source sensitivity than Parkes (nominally about $65 \%$, but it could be about $90 \%$ if the system temperature can be reduced to $35 \mathrm{~K}$ ), ASKAP can still achieve good signal-to-noise ratios on millisecond pulsars (MSPs) with sufficiently long integrations. It is in a low-interference environment and should be somewhat more robust to interference than a single dish. These properties could allow it to take on part of the Southern-hemisphere burden of monitoring MSPs for the purposes of contraints on a background of gravitational waves, and perhaps participate in an eventual detection [e.g., 7]. This would make ASKAP part of the International Pulsar Timing Array Project. While the MSPs are being monitored, the rest of the 30-square-degree ASKAP FOV need not go to waste; there are often other pulsars visible, and in the Galactic Plane there are regions where 20 or more pulsars are accessible at once (though not all of these contain MSPs). With new pulsars being discovered in ongoing surveys [e.g., 8], the surface density of sources will only increase. In general, we expect most of the "secondary" pulsars in the FOV to be young. Many may glitch or show large amounts of timing systematics [e.g., 9] and/or be targets for high-energy observations, for example with the Fermi Gamma-ray Observatory. Both young and millisecond pulsars are exciting targets for gravitational-wave observatories such as LIGO. There are therefore multiple motivations for ongoing monitoring to produce accurate radio ephemerides, and to record time-series data for pulsars known to glitch.

\section{Point-Source Searches}

The myriad science areas accessible to pulsar observations will all benefit from the discovery of previously unknown pulsars, either by finding unique objects and test-cases, or by the improved statistics of a larger and more comprehensive sample. Again, it is necessary to iron out the technical details of how to accomplish pulsar surveys with an interferometer, in order to fulfill part of one of the Key Science Projects of the SKA [10].

One technique aimed at discovering primarily millisecond and fast binary pulsars will be to search pulsar-like point sources found in the large continuum surveys such as EMU (Evolving Map of the Universe) and the transient surveys such as VAST (Variability and Slow Transients) and CRAFT (Commensal Real-time ASKAP Fast Transients) that will 
be carried out with ASKAP. EMU will find weak point sources, which we can examine for steep spectral indices and/or strong polarization, which may be signposts to pulsars. VAST and CRAFT will find stronger sources that are time- and/or frequency-variable; the scintillation properties may also indicate that pulsars are present. Work is underway to examine current point-source catalogs to determine the optimal criteria to use. Using tied-array beams on the point sources, we will be able to record fast-sampled, highfrequency-resolution data and thus be sensitive to millisecond pulsars. The selection effects will be different from those of traditional wide-area searches, possibly opening up an entirely new pulsar discovery space.

\section{Wide-Area Searches}

Once again aiming to develop techniques that will be needed for the SKA, we also plan to carry out a search of a wide area of sky, using the data recorded from the entire ASKAP FOV. Given the data rate limitations from the correlator, such a search will necessarily be restricted to comparatively wide frequency channels of a few $\mathrm{MHz}$ and to sample rates of milliseconds; even then the development of the necessary correlator modes will be some years off. The data reduction challenge is also significant, given the need to search either every pixel in the FOV or else the UV plane itself. We may choose to use only the inner core of 30 antennas to restrict the baselines to $2 \mathrm{~km}$ and reduce the number of pixels.

This search will of necessity be sensitive primarily to slower pulsars with spin periods greater than $\sim 10$ milliseconds, but there are a number of exciting types of objects in this category, including young pulsars, radio magnetars and even double-neutron-star binaries. To maximize the scientific output from such a survey, we intend to conduct searches in the lowest part of the ASKAP frequency band (700-1000 MHz, a regime not considered so far in wide-area searches) and a target zone just off the Galactic plane to minimize dispersion and scattering, while expecting both mildly recycled pulsars and young pulsars to be in our fields. Another likely target for such a survey will be the Magellanic Clouds, which we can cover deeply in just one pointing each.

\section{TECHNICAL DEVELOPMENTS AND PLANS}

Through the Design Study, we are investigating possible solutions to several algorithmic and hardware problems as well as planning trial observations with the BETA (Boolardy Engineering Test Array) prototype of 6 antennas that should be available in 2011-2.

Although the timing science plan is well-understood, one area that is being investigated is the optimal selection of particular fields on the sky that will place the most interesting pulsars near the centre of the FOV to minimize polarization impurities while still maintaining a good number of interesting secondary pulsars. Since we expect the list of primary pulsars to evolve as more objects are discovered, we need a reliable algorithm for FOV selection rather than a rigid list.

The point-source-search selection criteria are under active exploration using existing catalogs from the ATLAS survey [11]. Population syntheses for both the point-source 


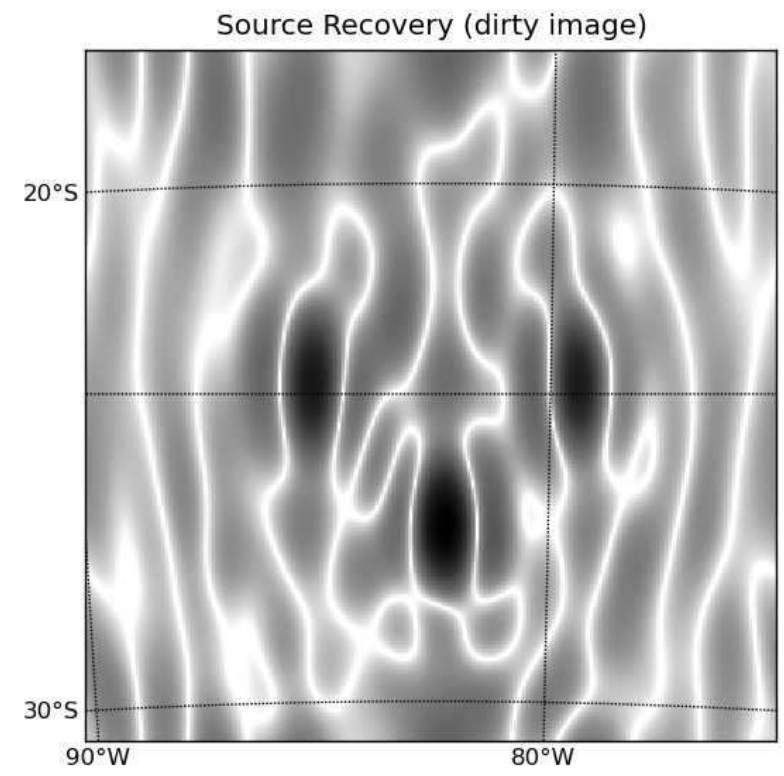

FIGURE 1. "Dirty image" showing recovery of simulated point sources. Visibility data was generated from an input sky with three equal-strength point sources, as observed by BETA tracking the southernmost source. The system temperature was set to 10 percent of the source flux.

and wide-area surveys are being carried out with two different code packages; see the article by Bouchard in this volume for details. Meanwhile, algorithm development for the wide-area searches is proceeding. Currently this includes simulation of both point-like and pulsed sources on the sky and software to detect pulsations along individual baselines. The next steps include searches of the simulated pixelized sky and development of an algorithm to indentify pulsar signals from unknown sky positions using multiple baselines simultaneously, effectively searching directly in the UV plane. The success of the intermediate stages of this software are shown in Figure 1, which demonstrates the successful recovery of simulated point sources.

We will need to provide pulsar backend hardware to acquire the signals and process them. This is made significantly easier relative to what is required at most telescopes by the ASKAP engineers' intention to provide us with tied-array beam signals that are already Nyquist-sampled and separated into few-MHz channels, or else in a full-Stokes digitized filterbank format. Therefore most of our data acquisition needs can be satisfied by computing power to handle the coherent dedispersion task for MSP timing, to fold or make dedispersed timeseries data for the slower pulsars and simply to record the filterbank data for the point-source searches. Disk space will be the main requirement for the last type of data, while small (possibly GPU-based) clusters should be able to handle the computational tasks. The wide-area survey will require significantly more and faster disks; this problem will be addressed closer to the time when these observations might be scheduled.

Our observations with the 6-element BETA telescope will focus on timing several 
bright pulsars simultaneously. In particular, we intend to observe two pulsars separated across the field of view to characterize the stability of the telescope response and tiedarray beams in particular over data spans of several hours.

\section{ACKNOWLEDGMENTS}

The Murchison Radio-astronomy Observatory (MRO) is jointly funded by the Commonwealth Government of Australia and State Government of Western Australia and managed by the CSIRO. We acknowledge the Wajarri Yamatji people as the traditional owners of the Observatory site. Pulsar and ASKAP research at UBC is supported by NSERC Discovery and SRO grants.

\section{REFERENCES}

1. D. R. Deboer, R. G. Gough, J. D. Bunton, T. J. Cornwell, R. J. Beresford, S. Johnston, I. J. Feain, A. E. Schinckel, C. A. Jackson, M. J. Kesteven, A. Chippendale, G. A. Hampson, J. D. O’Sullivan, S. G. Hay, C. E. Jacka, T. W. Sweetnam, M. C. Storey, L. Ball, and B. J. Boyle, IEEE Proceedings 97, 1507-1521 (2009).

2. S. G. Hay, J. D. O’Sullivan, J. S. Lot, C. Granet, A. Grancea, A. R. Forsyth, and D. H. Hayman, "Focal Plane Array Development for ASKAP (Australian SKA Pathfinder)," in Proc. EUCAP'07, 2007.

3. C. Carilli, and S. Rawlings, astro-ph/0409274 (2004).

4. N. Gupta, S. Johnston, I. Feain, and T. Cornwell (2008), CSIRO internal document, available at http://www.atnf.csiro.au/SKA/newdocs/configs-3.pdf.

5. S. Johnston, M. Bailes, N. Bartel, C. Baugh, M. Bietenholz, C. Blake, R. Braun, J. Brown, S. Chatterjee, J. Darling, A. Deller, R. Dodson, P. G. Edwards, R. Ekers, S. Ellingsen, I. Feain, B. M. Gaensler, M. Haverkorn, G. Hobbs, A. Hopkins, C. Jackson, C. James, G. Joncas, V. Kaspi, V. Kilborn, B. Koribalski, R. Kothes, T. L. Landecker, E. Lenc, J. Lovell, J. Macquart, R. Manchester, D. Matthews, N. M. McClure-Griffiths, R. Norris, U. Pen, C. Phillips, C. Power, R. Protheroe, E. Sadler, B. Schmidt, I. Stairs, L. Staveley-Smith, J. Stil, R. Taylor, S. Tingay, A. Tzioumis, M. Walker, J. Wall, and M. Wolleben, Proc. Astr. Soc. Aust. 24, 174-188 (2007).

6. S. Johnston, R. Taylor, M. Bailes, N. Bartel, C. Baugh, M. Bietenholz, C. Blake, R. Braun, J. Brown, S. Chatterjee, J. Darling, A. Deller, R. Dodson, P. Edwards, R. Ekers, S. Ellingsen, I. Feain, B. Gaensler, M. Haverkorn, G. Hobbs, A. Hopkins, C. Jackson, C. James, G. Joncas, V. Kaspi, V. Kilborn, B. Koribalski, R. Kothes, T. Landecker, A. Lenc, J. Lovell, J. Macquart, R. Manchester, D. Matthews, N. McClure-Griffiths, R. Norris, U. Pen, C. Phillips, C. Power, R. Protheroe, E. Sadler, B. Schmidt, I. Stairs, L. Staveley-Smith, J. Stil, S. Tingay, A. Tzioumis, M. Walker, J. Wall, and M. Wolleben, Experimental Astronomy 22, 151-273 (2008).

7. F. A. Jenet, G. B. Hobbs, K. J. Lee, and R. N. Manchester, ApJ 625, L123-L126 (2005).

8. M. J. Keith, A. Jameson, W. van Straten, M. Bailes, S. Johnston, M. Kramer, A. Possenti, S. D. Bates, N. D. R. Bhat, M. Burgay, S. Burke-Spolaor, N. D’Amico, L. Levin, P. L. McMahon, S. Milia, and B. W. Stappers, MNRAS 409, 619-627 (2010).

9. A. Lyne, G. Hobbs, M. Kramer, I. Stairs, and B. Stappers, Science 329, 408-412 (2010).

10. M. Kramer, D. C. Backer, J. M. Cordes, T. J. W. Lazio, B. W. Stappers, and S. Johnston, New Astr. 48, 993-1002 (2004).

11. R. P. Norris, J. Afonso, P. N. Appleton, B. J. Boyle, P. Ciliegi, S. M. Croom, M. T. Huynh, C. A. Jackson, A. M. Koekemoer, C. J. Lonsdale, E. Middelberg, B. Mobasher, S. J. Oliver, M. Polletta, B. D. Siana, I. Smail, and M. A. Voronkov, AJ 132, 2409-2423 (2006). 